\title{
Design of Large Scale Decentralized Low-Order Robust Control System Stabilizer to Mitigate the Damping of the System
}

\author{
Sharad Chandra Rajpoot ${ }^{1}$, Prashant Singh Rajpoot ${ }^{2}$, kishan Gupta ${ }^{3}$ Durga \\ Sharma ${ }^{4}$ \\ ${ }^{l}$ Assistant professor G.E.C. Jagdalpur, bastar, Chhattisgarh, India, \\ ${ }^{2}$ Assistant professor L.C.I.T., Bilaspur, Chhattisgarh, India, \\ ${ }^{3}$ electrical instructor aadimjati Kalyan Vibhag, Bilaspur, Chhattisgarh, India, \\ ${ }^{4}$ Assistant professor C.V.R.U. Kota, Bilaspur, Chhattisgarh, India,
}

\begin{abstract}
A discrete system is a system with a countable number of states. Discrete systems may be contrasted with continuous systems, which may also be called analog systems. A final discrete system is often modeled with a directed graph and is analyzed for correctness and complexity according to computational theory. Because discrete systems have a countable number of states, they may be described in precise mathematical models. A computer is a finite state machine that may be viewed as a discrete system. Because computers are often used to model not only other discrete systems but continuous systems as well, methods have been developed to represent real-world continuous systems as discrete systems. One such method involves sampling a continuous signal at discrete time intervals.Statistical process control (SPC) is a method of quality control which uses statistical methods. SPC is applied in order to monitor and control a process. Monitoring and controlling the process ensures that it operates at its full potential. At its full potential, the process can make as much conforming product as possible with a minimum (if not an elimination) of waste (rework or scrap). SPC can be applied to any process where the "conforming product" (product meeting specifications) output can be measured. Key tools used in SPC include control charts; a focus on continuous improvement; and the design of experiments. An example of a process where SPC is applied is manufacturing lines.

Index Terms: Statistical process control (SPC), Discrete system, quality control, discrete event system (DES), Damping, large scale decentralized control.
\end{abstract}

\section{Introduction}

By combining the knowledge gained from an analysis of the biophysical properties of natural antibody variable domains, the effects of mutations obtained in directed evolution experiments, and the detailed structural comparison of antibodies, it has now become possible to engineer antibodies for higher thermodynamic stability and more efficient folding. This is particularly important when antibodies are to be used under conditions where the disulfide bonds cannot form, i.e., in intracellular applications (as "intrabodies"). We describe in detail two methods for the knowledge-based improvement of antibody stability and folding efficiency. While CDR grafting from a non-human to the most closely related human antibody framework is an established technique to reduce the immunogenicity of a therapeutic antibody, CDR grafting for stabilization implies the use of a more distantly related acceptor framework with superior biophysical characteristics. The use of such dissimilar frameworks requires particular attention to antigen contact residues outside the classical CDR definition and to residues capable of indirectly affecting the conformation of the antigen binding site. As a second alternative, the stability of a suboptimal framework can be improved by the introduction of point mutations designed to optimize key residue interactions. We describe the analysis methods used to identify such point mutations, which can be introduced all at once, while maintaining the framework features necessary for antigen binding. These rational approaches render the continued "rediscovery" of certain mutations by directed evolution unnecessary, but they can also be used in conjunction with such methods to discover even better molecules

The examples thus far have shown continuous time systems and control solutions. In fact, as optimal control solutions are now often implemented digitally, contemporary control theory is now primarily concerned with discrete time systems and solutions. The Theory of Consistent Approximations ${ }^{[19]}$ provides conditions under which solutions to a series of increasingly accurate discredited optimal control problem converge to the solution of the original, continuous-time problem. Not all discretization methods have this property, even seemingly obvious ones. For instance, using a variable step-size routine to integrate the problem's dynamic equations may generate a gradient which does not converge to zero (or point in the right direction) as the solution is approached. The direct method RIOTS is based on the Theory of Consistent Approximation. 


\subsection{Motivatition And Objective}

Optimal control theory, an extension of the calculus of variations, is a mathematical optimization method for deriving control policies. The method is largely due to the work of Lev Pontryagin and Richard Bellman in the 1950s, after contributions to calculus of variations by Edward J. McShane. Optimal control can be seen as a control strategy in control theory.[5] A discrete event system (DES) is a dynamic system that evolves in accordance with the abrupt occurrence, at possibly unknown irregular intervals, of physical events. Such systems arise in a variety of contexts ranging from computer operating systems to the control of complex multimode processes. A control theory for the logical aspects of such DESs is surveyed. The focus is on the qualitative aspects of control, but computation and the related issue of computational complexity are also considered. Automata and formal language models for DESs are surveyed.

\section{1overview}

\section{Damping}

A decreasing of the amplitude of an electrical or mechanical wave. an energy-absorbing mechanism or resistance circuit causing this decrease. a reduction in the amplitude of an oscillation or vibration as a result of energy being dissipated as heat. Damping is a reduction in vibration over a period of time.[3] Damping will eventually bring a vibrating object to rest. damping mechanism suppresses vibrations of the structure. Damping is a reduction in vibration over a period of time.

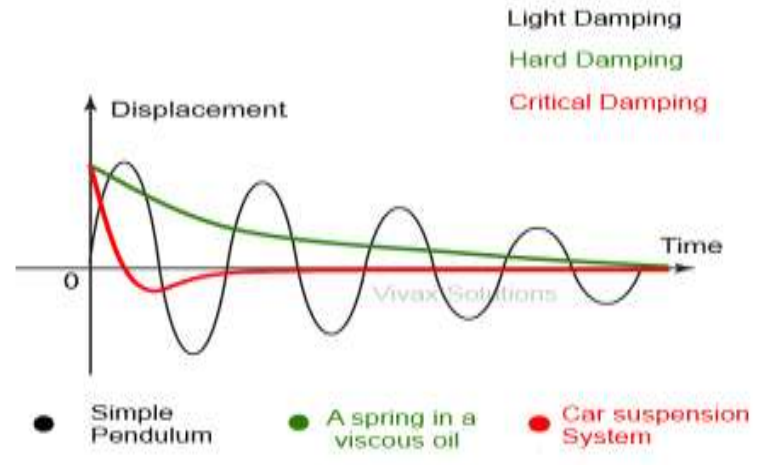

Fig. damping types

\subsection{Damping ratio}

In engineering, the damping ratio is a dimensionless measure describing how oscillations in a system decay after a disturbance. Many systems exhibit oscillatory behavior when they are disturbed from their position of static equilibrium. A mass suspended from a spring, for example, might, if pulled and released, bounce up and down. On each bounce, the system is "trying" to return to its equilibrium position, but overshoots it. Sometimes losses (e.g. frictional) damp the system and can cause the oscillations to gradually decay in amplitude towards zero or attenuate. [12]The damping ratio is a measure of describing how rapidly the oscillations decay from one bounce to the next. The behavior of oscillating systems is often of interest in a diverse range of disciplines that include control engineering, mechanical engineering, structural engineering, and electrical engineering. The physical quantity that is oscillating varies greatly, and could be the swaying of a tall building in the wind, or the speed of an electric motor, but a normalized, or non-dimensionalised approach can be convenient in describing common aspects of behavior.[11]

\subsection{Oscillation case}

- Where the spring- mass system is completely lossless, the mass would oscillate indefinitely, with each bounce of equal height to the last. This hypothetical case is called undamped.

- If the system contained high losses, for example if the spring- mass experiment were conducted in a viscous fluid, the mass could slowly return to its rest position without ever overshooting. This case is called overdamped.

- Commonly, the mass tends to overshoot its starting position, and then return, overshooting again. With each overshoot, some energy in the system is dissipated, and the oscillations die towards zero. This case is called under damped.

- Between the over damped and under damped cases, there exists a certain level of damping at which the system will just fail to overshoot and will not make a single oscillation.[13] This case is called critical damping. The key difference between critical damping and over damping is that, in critical damping, the system returns to equilibrium in the minimum amount of time. 


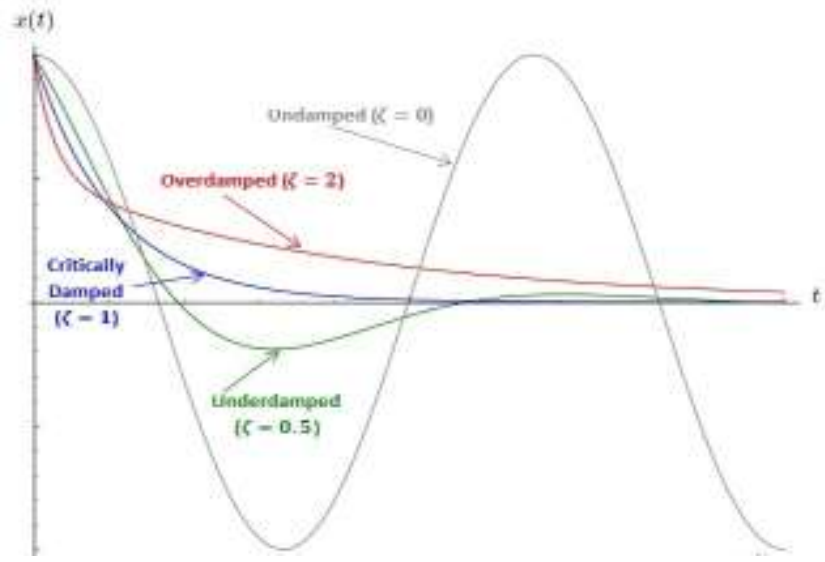

Fig. damping cases

\subsection{Definition}

The damping ratio is a parameter, usually denoted by $\zeta$ (zeta),[11]that characterizes the frequency response of a second order ordinary differential equation. It is particularly important in the study of control theory. It is also important in the harmonic oscillator. The damping ratio provides a mathematical means of expressing the level of damping in a system relative to critical damping. For a damped harmonic oscillator with mass $m$, damping coefficient $c$, and spring constant $k$, it can be defined as the ratio of the damping coefficient in the system's differential equation to the critical damping coefficient:

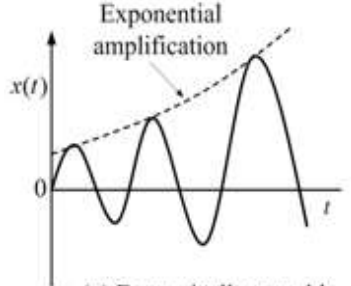

(a) Dynamically unstable

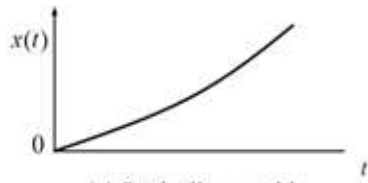

(c) Statically unstable

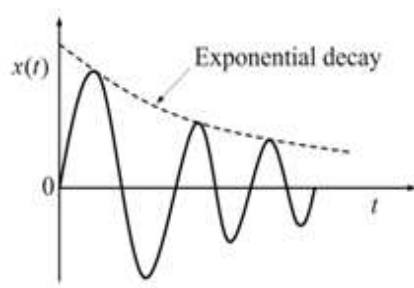

(b) Dynamically stable

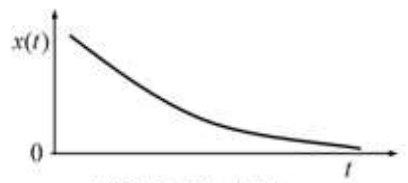

(d) Statically stable

\section{1 overview}

\section{Proportional- Integral- Derivative Pid Controller}

PID controller) is a control loop feedback mechanism (controller) commonly used in industrial control systems. APID controller continuously calculates an error value as the difference between a desired setp point and a measured process variable and applies a correction based on proportional, integral, and derivative terms (sometimes denoted $P, I$, and $D$ respectively) which give their name to the controller type.

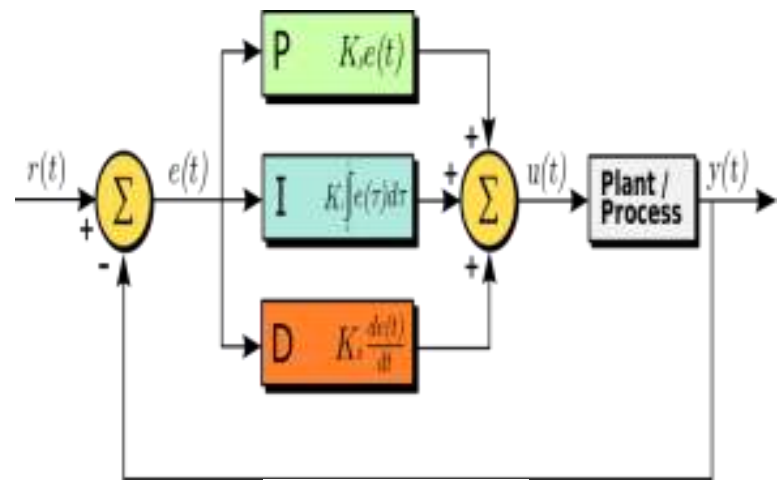

Fig. PID controller 
where $\mathrm{K}_{\mathrm{p}}, \mathrm{K}_{\mathrm{d}}, \mathrm{K}_{\mathrm{i}}$ and all non-negative, denote the coefficients for the proportional, integral, and derivative terms, respectively (sometimes denoted $P, I$, and $D$ ). In this model:

- $\quad P$ accounts for present values of the error. For example, if the error is large and positive, the control output will also be large and positive.

- I accounts for past values of the error. For example, if the current output is not sufficiently strong, the integral of the error will accumulate over time, and the controller will respond by applying a stronger action.

- $D$ accounts for possible future trends of the error, based on its current rate of change. [9]

As a PID controller relies only on the measured process variable, not on knowledge of the underlying process, it is broadly applicable. By tuning the three parameters of the model, a PID controller can deal with specific process requirements. The response of the controller can be described in terms of its responsiveness to an error, the degree to which the system overshoots a setpoint, and the degree of any system oscillation. The use of the PID algorithm does not guarantee optimal control of the system or even its stability. Some applications may require using only one or two terms to provide the appropriate system control. This is achieved by setting the other parameters to zero. A PID controller is called a PI, PD, P or I controller in the absence of the respective control actions. PI controllers are fairly common, since derivative action is sensitive to measurement noise, whereas the absence of an integral term may prevent the system from reaching its target value. For discrete-time systems, the term PSD (proportional-summation-difference) is often used. [13]

\subsection{Manual tuning}

If the system must remain online, one tuning method is to first set $\mathrm{Ki}$ and $\mathrm{K}_{\mathrm{d}}$ values to zero. Increase the until the output of the loop oscillates, then the Kp should be set to approximately half of that value for a "quarter amplitude decay" type response. Then increase $K_{d}$ until any offset is corrected in sufficient time for the process. However, too much $\mathrm{K}_{\mathrm{d}}$ will cause instability. Finally, increase $\mathrm{K}_{\mathrm{d}}$, if required, until the loop is acceptably quick to reach its reference after a load disturbance. However, too much will cause excessive response and overshoot.[10] A fast PID loop tuning usually overshoots slightly to reach the setpoint more quickly; however, some systems cannot accept overshoot, in which case an over-damped closed-loop system is required, which will require a Kp setting significantly less than half that of the $\mathrm{Kp}$ setting that was causing oscillation.

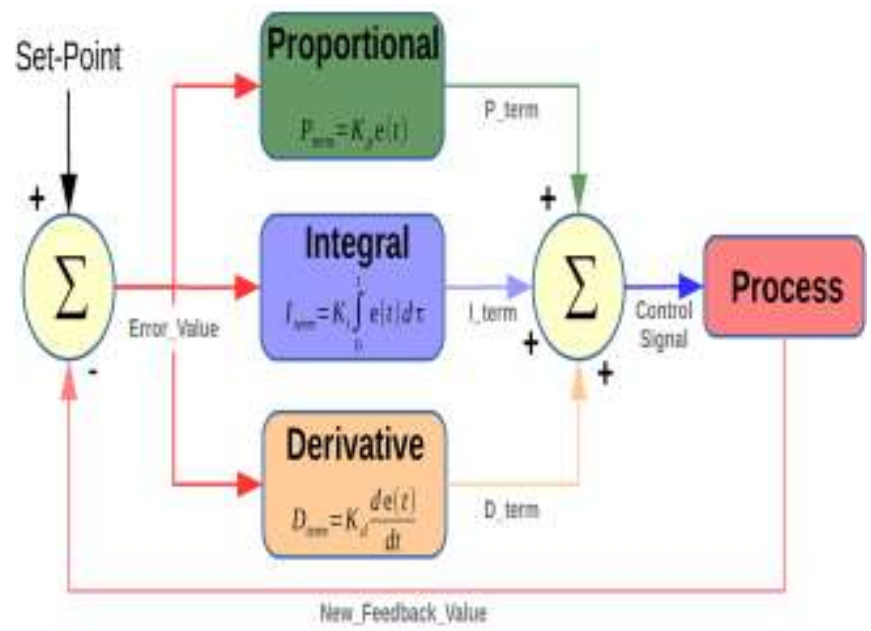

Fig. PID Manual tuning

\section{Controller-Design}

Consider the following vehicle control problem. There are $\mathrm{N}$ vehicles in a line, with vehicle i located at position qi, Suppose each vehicle is displaced a distance xi from its original unit spacing. Each vehicle has sensors which measure the relative displacements of its neighbors plus noise; for example, $y 2=x 3-x 2+w 2$ The proposed method of design of a controller by process reduction technique involves the following steps:

Step-1 Reduce the given higher order discrete system to a lower order model by error minimization technique. Step-2 Design a PID controller for the reduced ordersystem. The parameters of the PID controller are optimized using the same error same errorminimization technique employing RCGA.

Step-3 Test the designed PID controller for the reduced order model for which the PID controller has been, designed.

Step-4Test the designed PID controller for the original higher order model 
4.1 Static Proble

Let us return to the vehicle spacing problem considered Suppose there are 4 vehicles, and 3 sensor outputs

$$
\begin{aligned}
& \mathrm{y} 1=\mathrm{x} 2-\mathrm{x} 1+\mathrm{w} 1 \\
& \mathrm{y} 2=\mathrm{x} 3-\mathrm{x} 2+\mathrm{w} 2 \\
& \mathrm{y} 3=\mathrm{x} 4-\mathrm{x} 3+\mathrm{w} 3
\end{aligned}
$$

Let us restrict our attention to finding the best linear policy for the cost function, where each vehicle only receives information about its neighbors. We will later show that such a linear policy is in fact optimal. Linear controllers correspond to matrices $\mathrm{K}$, such that

$$
\mathrm{u}=\mathrm{Ky}
$$

\begin{tabular}{|l|l|l|l|l|l|}
\hline Para meter & Rise time & Overshoot & Settling time & Steady-state error & Stability \\
\hline Kp & Decrease & Increase & Small change & Decrease & Degrade \\
\hline \multicolumn{1}{|c|}{ Ki } & Decrease & Increase & Increase & Eliminate & Degrade \\
\hline Kd & Minor change & Decrease & Decrease & No effect in theory & Improve if small \\
\hline
\end{tabular}

\section{Decentrlised Control System}

Centralization of authority means the power of planning and decision making are exclusively in the hands of top management while in the case of Decentralization, the powers for the same has been disseminated by the top management to the middle or low-level management. There is a never ending debate between these two terms to prove which one is better. In this article,significant differences between Centralization and Decentralization, in an organisation is explained.
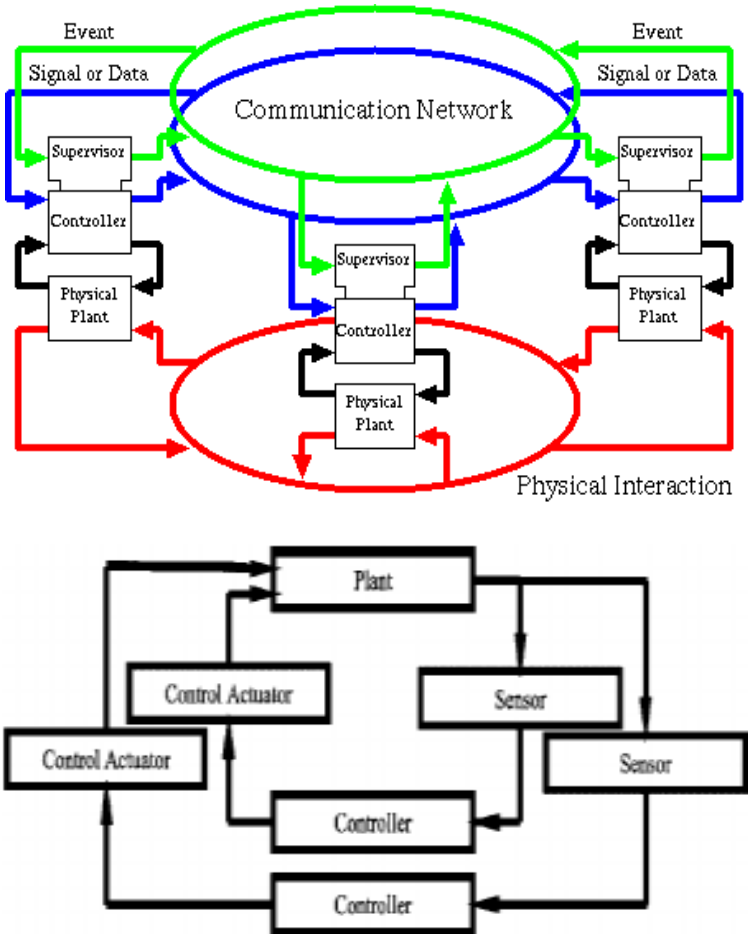

Fig. decentralized control system

\section{Real Coaded Genetic Algorithm}

In computer science and operations research, a genetic algorithm (GA) is a met heuristic inspired by the process of natural selection that belongs to the larger class of evolutionary algorithms (EA). Genetic algorithms are commonly used to generate high-quality solutions to optimization and search problems by relying on bioinspired operators such as mutation, crossover and selection

\subsection{Optimization problems}

In a genetic algorithm, a population of candidate solutions (called individuals, creatures, or phenotypes) to an optimization problem is evolved toward better solutions. Each candidate solution has a set of properties (its chromosomes or genotype) which can be mutated and altered; traditionally, solutions are represented in binary as strings of $0 \mathrm{~s}$ and $1 \mathrm{~s}$, but other encodings are also possible. ${ }^{[2]}$ The evolution usually starts from a population of randomly generated individuals, and is an iterative process, with the population in each 
iteration called a generation. In each generation, the fitness of every individual in the population is evaluated; the fitness is usually the value of the objective function in the optimization problem being solved. The more fit individuals are stochastically selected from the current population, and each individual's genome is modified (recombined and possibly randomly mutated) to form a new generation. The new generation of candidate solutions is then used in the next iteration of the algorithm. Commonly, the algorithm terminates when either a maximum number of generations has been produced, or a satisfactory fitness level has been reached for the population.

A typical genetic algorithm requires:

1. a genetic representation of the solution domain,

2. a fitness function to evaluate the solution domain.

Once the genetic representation and the fitness function are defined, a GA proceeds to initialize a population of solutions and then to improve it through repetitive application of the mutation, crossover, inversion and selection operators.

\section{Termination}

This generational process is repeated until a termination condition has been reached. Common terminating conditions are:

- A solution is found that satisfies minimum criteria

- Fixed number of generations reached

- Allocated budget (computation time/money) reached

- The highest ranking solution's fitness is reaching or has reached a plateau such that successive iterations no longer produce better results

- Manual inspection

- Combinations of the above

\subsection{Chromosome representation}

The simplest algorithm represents each chromosome as a bit string. Typically, numeric parameters can be represented by integers, though it is possible to use floating point representations. The floating point representation is natural to evolution strategies and evolutionary programming. The notion of real-valued genetic algorithms has been offered but is really a misnomer because it does not really represent the building block theory that was proposed by John Henry Holland in the 1970s. This theory is not without support though, based on theoretical and experimental results (see below). The basic algorithm performs crossover and mutation at the bit level. Other variants treat the chromosome as a list of numbers which are indexes into an instruction table, nodes in a linked list, hashes, objects, or any other imaginable data structure. Crossover and mutation are performed so as to respect data element boundaries. For most data types, specific variation operators can be designed. Different chromosomal data types seem to work better or worse for different specific problem domains.

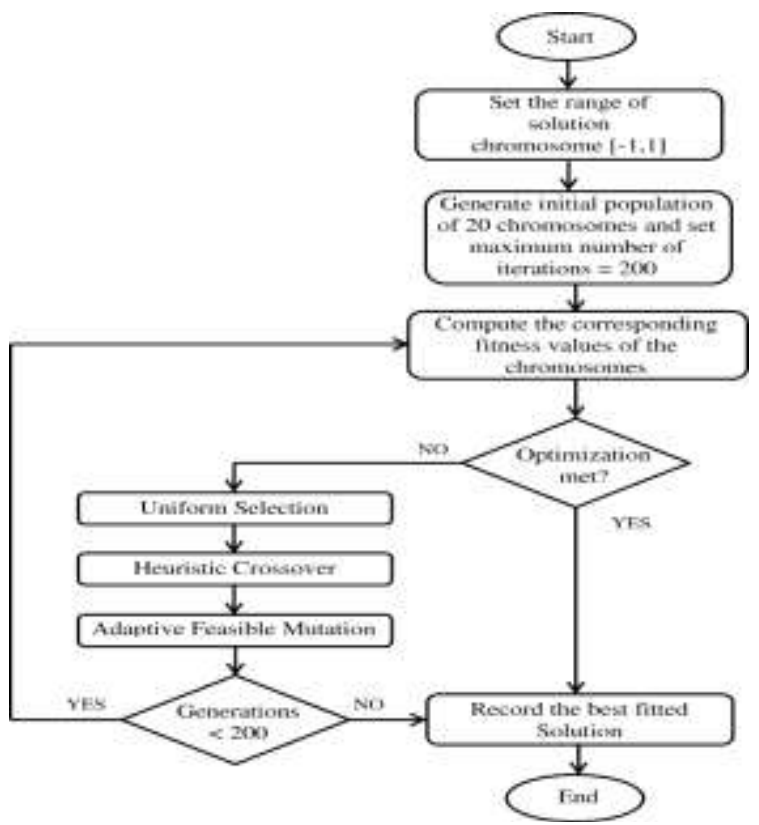

Fig. Chromosome representation 
When bit-string representations of integers are used, Gray coding is often employed. In this way, small changes in the integer can be readily affected through mutations or crossovers. This has been found to help prevent premature convergence at so called Hamming walls, in which too many simultaneous mutations (or crossover events) must occur in order to change the chromosome to a better solution.

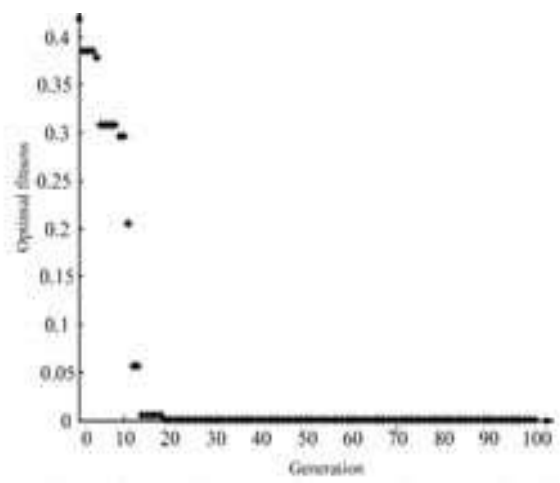

Fig. Convergence of fitness function
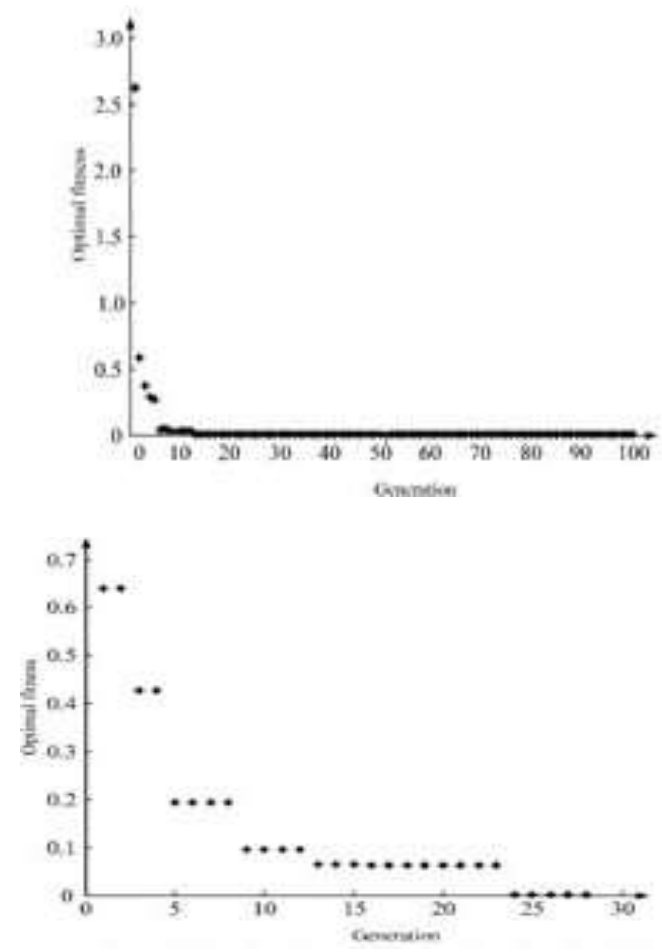

Fig. Optimized results of the improved real coded genetic algorithm

\section{VII.Results}

Closed loop the simplest algorithm represents each chromosome controller

\begin{tabular}{|l|l|}
\hline$-6.1328 \mathrm{e}-01+6.129 \mathrm{e}-01 \mathrm{i}$ & $-2.9519 \mathrm{e}-07+6.309 \mathrm{e}-02 \mathrm{i}$ \\
\hline$-5.1328 \mathrm{e}-01-6.129 \mathrm{e}-01 \mathrm{i}$ & $-3.9399 \mathrm{e}-07-7.309 \mathrm{e}-02 \mathrm{i}$ \\
\hline$-2.5272 \mathrm{e}-01+4.437 \mathrm{e}-01 \mathrm{i}$ & $\mathbf{4 . 7 4 3 0 e - 0 5}+\mathbf{1 . 6 9 6 e - 0 2 i}$ \\
\hline$-2.5272 \mathrm{e}-01-3.547 \mathrm{e}-01 \mathrm{i}$ & $\mathbf{5 . 7 4 3 0 e - 0 5}-\mathbf{4 . 6 9 6 e - 0 2 i}$ \\
\hline$-2.2851 \mathrm{e}-01+5.107 \mathrm{e}-01 \mathrm{i}$ & $-1.2791 \mathrm{e}-03+5.604 \mathrm{e}-02 \mathrm{i}$ \\
\hline$-2.2851 \mathrm{e}-01-3.107 \mathrm{e}-01 \mathrm{i}$ & $-1.2791 \mathrm{e}-03-7.604 \mathrm{e}-02 \mathrm{i}$ \\
\hline$-2.2081 \mathrm{e}-01+3.855 \mathrm{e}-01 \mathrm{i}$ & $\mathbf{3 . 3 0 0 3 e - 0 4}+\mathbf{5 . 3 5 6 e - 0 2 i}$ \\
\hline$-2.2081 \mathrm{e}-01-3.855 \mathrm{e}-01 \mathrm{i}$ & $\mathbf{2 . 3 0 1 2 e - 0 4}-\mathbf{4 . 3 5 6 e - 0 2 i}$ \\
\hline$-5.0705 \mathrm{e}-01+0.000 \mathrm{e}+00 \mathrm{i}$ & $-1.5670 \mathrm{e}-03+4.793 \mathrm{e}-02 \mathrm{i}$ \\
\hline$-5.7014 \mathrm{e}-01+0.001 \mathrm{e}+01 \mathrm{i}$ & $-1.5670 \mathrm{e}-03-4.793 \mathrm{e}-02 \mathrm{i}$ \\
\hline$-4.4214 \mathrm{e}-01+0.000 \mathrm{e}+00 \mathrm{i}$ & $\mathbf{3 . 4 4 9 8 e - 0 5}+\mathbf{4 . 5 0 1 e - 0 2 i}$ \\
\hline$-2.5548 \mathrm{e}-01+2.626 \mathrm{e}-01 \mathrm{i}$ & $\mathbf{4 . 5 6 9 8 e - 0 5}-\mathbf{3 . 5 0 1 e - 0 2 i}$ \\
\hline$-2.5332 \mathrm{e}-01-2.626 \mathrm{e}-01 \mathrm{i}$ & $-4.1667 \mathrm{e}-03+3.547 \mathrm{e}-02 \mathrm{i}$ \\
\hline
\end{tabular}




\begin{tabular}{|l|l|}
\hline$-2.3143 \mathrm{e}-01+2.390 \mathrm{e}-01 \mathrm{i}$ & $-3.1587 \mathrm{e}-03-3.937 \mathrm{e}-02 \mathrm{i}$ \\
\hline$-2.4254 \mathrm{e}-04+7.327 \mathrm{e}-02 \mathrm{i}$ & $\mathbf{4 . 8 4 4 3 3 e - 0 4}+\mathbf{3 . 2 5 7 e - 0 2 i}$ \\
\hline$-2.0645 \mathrm{e}-01+2.111 \mathrm{e}-01 \mathrm{i}$ & $\mathbf{4 . 4 3 3 6 e - 0 4} \mathbf{- 3 . 2 5 7 e - 0 2 i}$ \\
\hline$-2.0645 \mathrm{e}-01-2.117 \mathrm{e}-01 \mathrm{i}$ & $-8.0886 \mathrm{e}-04+2.452 \mathrm{e}-02 \mathrm{i}$ \\
\hline$-8.7694 \mathrm{e}-02+1.752 \mathrm{e}-01 \mathrm{i}$ & $-8.0506 \mathrm{e}-04-2.652 \mathrm{e}-02 \mathrm{i}$ \\
\hline$-9.7694 \mathrm{e}-02-1.752 \mathrm{e}-01 \mathrm{i}$ & $-6.3742 \mathrm{e}-05+8.236 \mathrm{e}-03 \mathrm{i}$ \\
\hline$-1.9589 \mathrm{e}-01+0.000 \mathrm{e}+00 \mathrm{i}$ & $-7.2842 \mathrm{e}-05-6.236 \mathrm{e}-03 \mathrm{i}$ \\
\hline$-5.3731 \mathrm{e}-02+7.002 \mathrm{e}-02 \mathrm{i}$ & $\mathbf{0 . 0 0 0 0 e}+\mathbf{0 0}+\mathbf{0 . 0 0 0 e}+\mathbf{0 0} \mathbf{i}$ \\
\hline$-5.3731 \mathrm{e}-02-7.002 \mathrm{e}-02 \mathrm{i}$ & $\mathbf{7 . 8 6 4 0 e - 0 6}+\mathbf{2 . 0 3 7 e - 0 2 i}$ \\
\hline$-8.4833 \mathrm{e}-04+7.230 \mathrm{e}-02 \mathrm{i}$ & $\mathbf{7 . 8 6 4 0 e - 0 6} \mathbf{- 2 . 0 3 7 e - 0 2 i}$ \\
\hline$-8.4833 \mathrm{e}-04-7.230 \mathrm{e}-02 \mathrm{i}$ & $-1.3274 \mathrm{e}-04+2.050 \mathrm{e}-02 \mathrm{i}$ \\
\hline$-2.4254 \mathrm{e}-04+7.327 \mathrm{e}-02 \mathrm{i}$ & $-1.3274 \mathrm{e}-04-2.050 \mathrm{e}-02 \mathrm{i}$ \\
\hline$-2.4254 \mathrm{e}-04-7.327 \mathrm{e}-02 \mathrm{i}$ & $\mathbf{6 . 1 5 9 5 e - 0 6}+\mathbf{2 . 0 6 1 e - 0 2 i}$ \\
\hline $2.4254 \mathrm{e}-04+7.327 \mathrm{e}-02 \mathrm{i}$ & $-9.0546 \mathrm{e}-04+2.662 \mathrm{e}-02 \mathrm{i}$ \\
\hline
\end{tabular}

\section{Displacement}

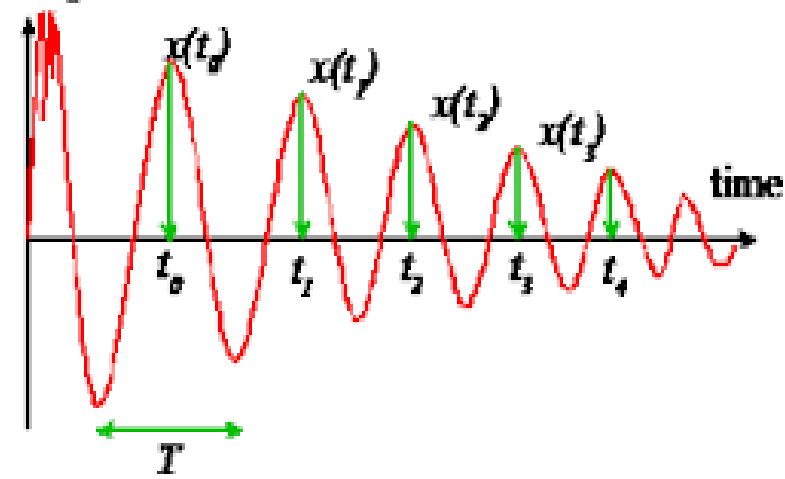

[1]

Displacement

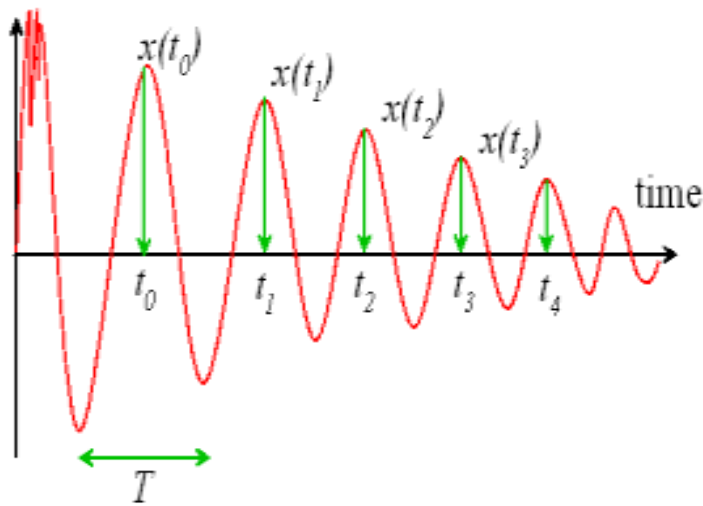

[2]

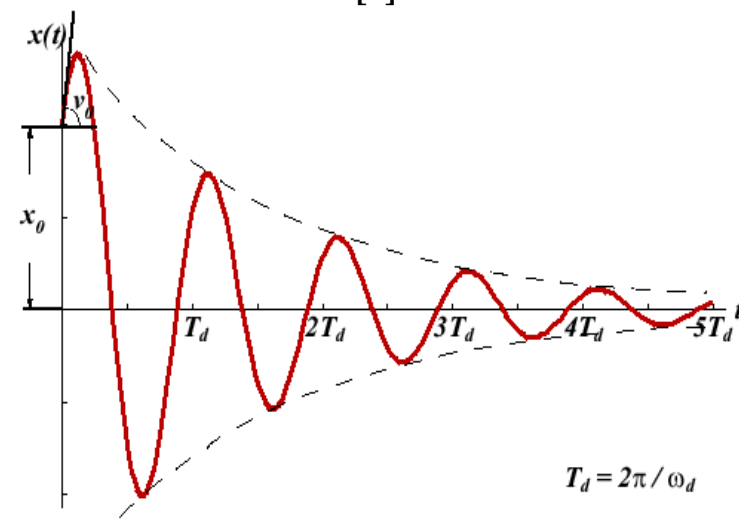

[3]

Fig. minor damping control 


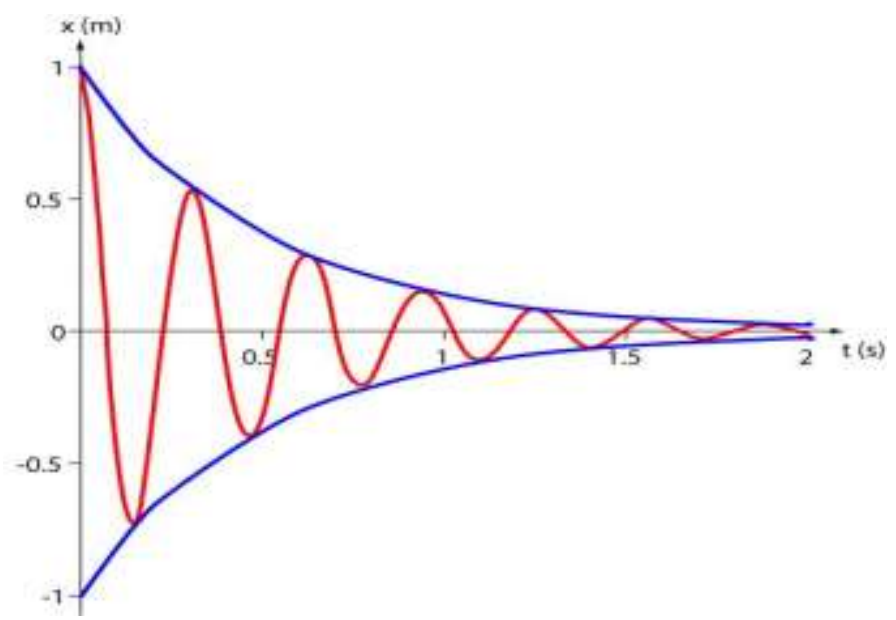

[4]

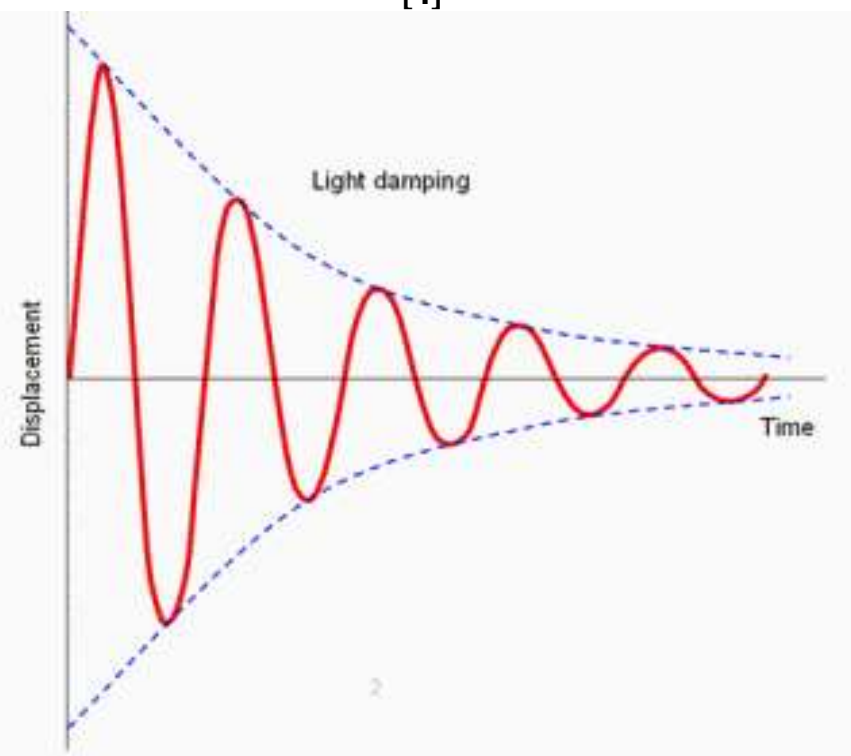

[5]

Fig. light damping stage

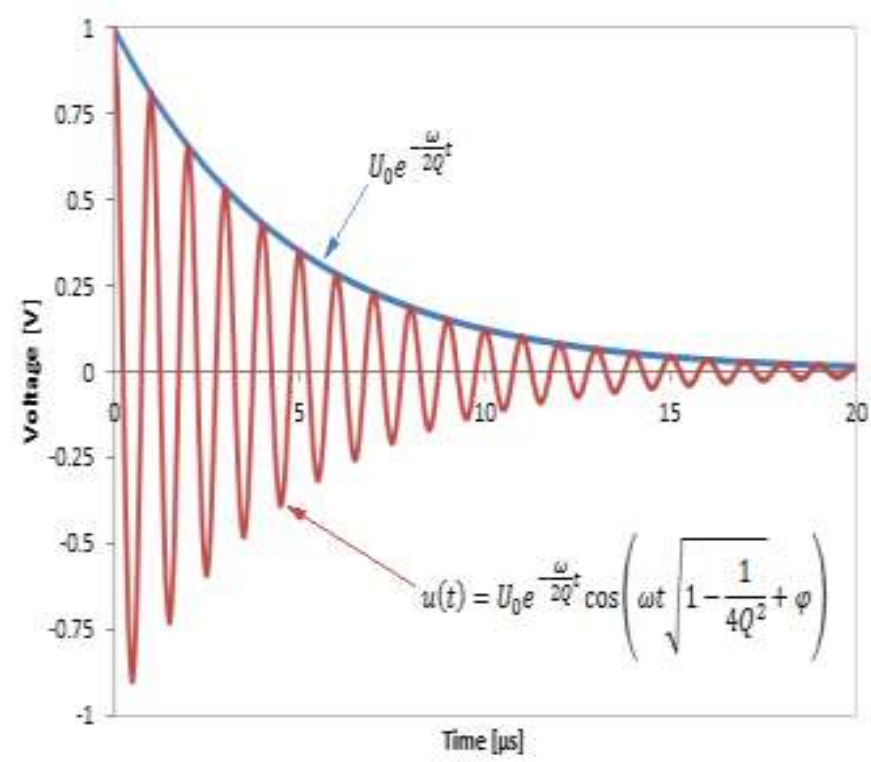




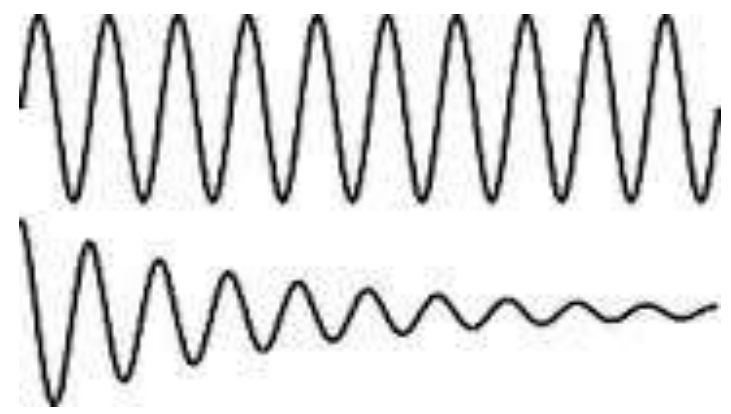

Fig. undamped and damped oscillation

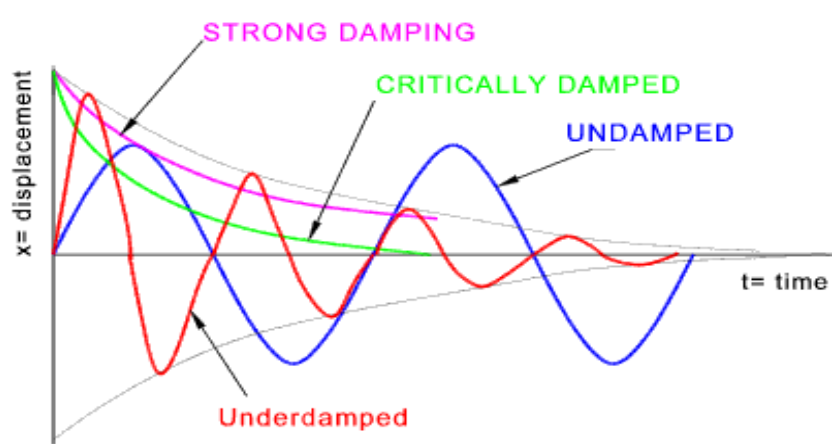

Fig. Damping analysis

\section{Conclusions}

This paper has demonstrated the effectiveness of the convex optimization in obtaining lower order robust damping controller for inter-area oscillation damping. This paper has proposed a new decentralized controller design method for large scale systems The proposed design achieves excellent performance using a low order controller. In fact, it is somewhat surprising that the proposed multivariable decentralized controller which has only 3 scalar parameters can produce better performance than the standard centralized LQR-observer. In particular, in this study, the LFSS plant model originally proposed in [13] had 5 inputs and 5 outputs and consisted of 100 state variables. Due to space limitations, this plant model was reduced to a 24th order system, and in this case, if a LQR observer controller was used, it would result in a 24th order controller, as compared to the 5th order proposed decentralized controller. If the original 100th order plant model was used, the centralized LQR-observer controller would now have a controller consisting of order 105, as compared to the present controller, which would still be only 5th order..

\section{References}

[1]. M.J.Balas, "Some approaches to the control of large space structures," A.A.S Annual Rocky Mountain Guidance and Contr. Conf.,May 1978.

[2]. $\quad$ R.E.Skelton and P.C.Hughes, "Modal truncation for flexible spacecraft," J.Guidance Contr, vol. 4, pp. 291-297, MayJune 1981

[3]. OZ.L. Meirovitch, "Modal-space control of large flexible spacecraft possessing ignorable coordinates," Proc. 2ndVPI and SU/AIAA Symp. on Dynamics and Contr. of large flexible Spacecraft, Blacksburg, VA, p. 45, June 1979.

[4]. M.J.Balas, "Modal control of certain flexible dynamic systems," SIAM J. Contr. Optimiz, vol. 16, pp. 450-462, May,1978.

[5]. I.Bar-Kana, R.Fischl and P.Kalata, "Direct Position Plus Velocity Feedback Control of Large Flexible Space Structures," Automatic Control, IEEE Transactions, pp. 1186-1188,1991

[6]. R.J.Benhabib, R.P.Iwens and R.L.Jackson, "Adaptive controlfor large space structures," Proc. 1979 Conf. Decision and Contr, pp. 214-217, 1979.

[7]. $\quad$ Y. Fujisaki, M. Ikeda and K. Miki, "Robust Stabilization of Large Space Structures via Displacement Feedback," Automatic Control, IEEE Transactions on, vol. 46 (12), pp. 1993-1996, Dec.2001.

[8]. S.M.Joshi, "Control of Large Flexible Space Structures.,"Lecture notes in control and information sciences, no. Springer-Verlag, 1998.

[9]. $\quad$ S.S.Ahmad, J.S.Lew and L.H.Keel, "Fault Tolerant Controller Design for Large space Structures," Control Applications, 1999. Proceeding of the 1999 IEEE International Conference, vol. 1, pp. 63-68, 1999

[10]. R. Shah, N. Mithulananthan, and R. C. Bansal, " Oscillatory stability analysis with high penetrations of large-scale photovoltaic generation,” Energy Conversion and Management, vol. 65, pp.420-429, Jan. 2013.

[11]. B. Pal and B. Chaudhuri, Robust Control in Power Systems, New York: Springer, 2005.

[12]. I. Kamwa, G. Trudel, and L. Gerin-Lajoie, " Robust design and coordination of multiple damping controllers using non-linear constraints optimization,” IEEE Trans. Power Systems, vol. 15, no. 3, pp. 1084- 1092, Sept. 2000.

[13]. R. V. de Oliveira, R. A. Ramos, and N. G. Bretas, " A mixed procedure based on classical and modern robust damping control," IEEE Trans. Power Systems, vol. 22, no. 3, pp. 1231-1239, Aug. 2007. 